\title{
The Classical Double Slit Interference Experiment: A New Geometrical Approach
}

\author{
Joseph Ivin Thomas ${ }^{1,2}$ \\ ${ }^{1}$ School of Natural Sciences \& Engineering, National Institute of Advanced Studies, IISc Campus, Bangalore, India \\ ${ }^{2}$ Department of Physiology, East Point Medical College \& Research Centre, Bangalore, India
}

\section{Email address:}

dr.ivinthomas@yahoo.com

\section{To cite this article:}

Joseph Ivin Thomas. The Classical Double Slit Interference Experiment: A New Geometrical Approach. American Journal of Optics and Photonics. Vol. 7, No. 1, 2019, pp. 1-9. doi: 10.11648/j.ajop.20190701.11

Received: March 2, 2019; Accepted: April 9, 2019; Published: May 7, 2019

\begin{abstract}
The double slit experiment was first conceived of by the English physician-physicist Thomas Young in 1801. It was the first demonstrative proof that light possesses a wave nature. In this experiment, light is made to pass through two very narrow slits that are spaced closely apart and a screen placed on the other side captures a pattern of alternating bright and dark stripes called fringes, formed as a result of the interference of ripples of light emanating from either slit. The relative positions and intensities of the fringes on the screen can be calculated by employing two assumptions that help simplify the geometry of the slit-screen arrangement. Firstly, the screen to slit distance is taken to be larger than the inter-slit distance (far field limit) and secondly, the inter-slit distance is taken to be larger than the wavelength of light. This conventional approach can account for the positions and intensities of the fringes located in the central portion of the screen with a fair degree of precision. It however, fails to account for those fringes located in the peripheral portions of the screen and also, is not applicable to the case wherein the screen to slit distance is made comparable to the inter-slit distance (near field limit). In this paper, the original analysis of Young's Experiment is reformulated using an analytically derived hyperbola equation, which is formed from the locus of the points of intersections of two uniformly expanding circular wavefronts of light that emanate from either slit source. Additionally, the shape of the screen used to capture the interference pattern is varied (linear, semicircular, semielliptical) and the relative positions of the fringes is calculated for each case. This new approach bears the distinctive advantage that it is applicable in both the far field and the near field scenarios, and since no assumptions are made beyond the Huygens-Fresnel principle, it is therefore, a much more generalized approach. For these reasons, the author suggests that the new analysis ought to be introduced into the Wave Optics chapter of the undergraduate Physics curriculum.
\end{abstract}

Keywords: Interference, Fringe, Hyperbola, Wavefront, Locus

\section{Introduction}

\subsection{Qualitative Aspects}

The double slit experiment was historically the first to decisively demonstrate and establish the wave nature of light, bringing to rest the then long-standing debate on whether light had a particle or a wave nature. [1,2] The apparatus used, consists of two barriers (see Figure 1). The first barrier has a single slit $\mathrm{S}$ and the second barrier placed just infront of the first, has two slits $S_{1}$ and $S_{2}$. Light in the form of a plane wave-front when incident on the first barrier, emerges out of $\mathrm{S}$ in the form of circular wave-fronts. Upon arrival at the second barrier, the single circular wave-front is split into two circular wave-fronts by slits $S_{1}$ and $S_{2} . S_{1}$ and $S_{2}$ behave as a pair of coherent light sources because the light waves emerging from them are derived from the same initial wave-front from $\mathrm{S}$ and therefore, bear a constant phase relationship. A viewing screen is situated some distance infront of the second barrier. Light from both slits $S_{1}$ and $S_{2}$ combine either constructively or destructively at various points on this screen, giving a visible pattern of alternating dark and bright parallel bands, called fringes. Constructive interference gives rise to a bright fringe and destructive interference to a dark fringe (see Figure 2). 


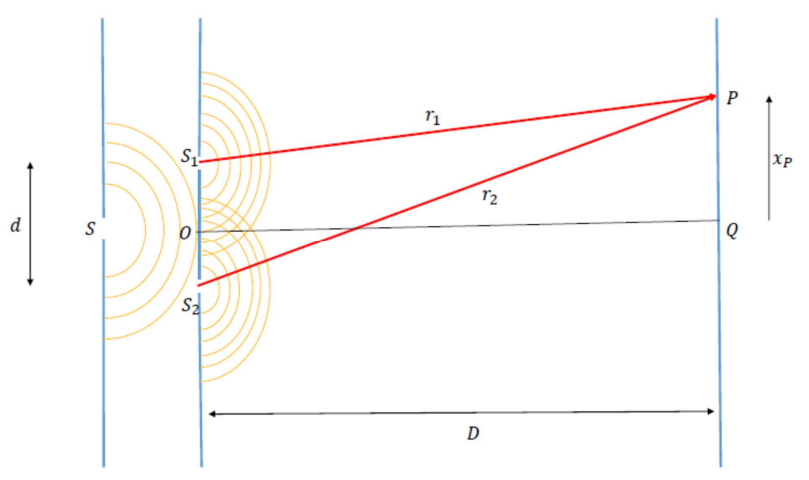

Figure 1. Arrangement of the Double Slit Apparatus.

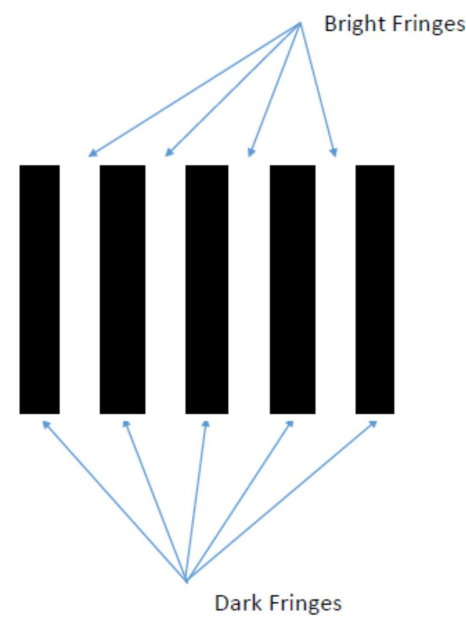

Figure 2. Interference Fringe Pattern.

\subsection{Quantitative Analysis}

Let the viewing screen be situated at a distance $D$ from the double slit barrier, the distance between the two slits $\mathrm{S}_{1}$ and $\mathrm{S}_{2}$ be $d$ and the wavelength of monochromatic light used be $\lambda$. Wavefronts emanating from $\mathrm{S}_{1}$ and $\mathrm{S}_{2}$ traverse distances $r_{1}$ and $r_{2}$ respectively, to reach an arbitrary point $\mathrm{P}$ on the distant screen. The disparity in the distances traversed $\left(=r_{2}-r_{1}\right)$ is called the path difference and is denoted by $\delta$. The standard formula for $\delta$ that can be found in many undergraduate textbooks is as follows [3-6]:

$$
\delta=r_{2}-r_{1}=d \cdot \sin \theta
$$

Where $\theta$ is the angle shown in Figure 3 .

The calculation of $\delta$ is based on two assumptions, that help simplify the geometry of the arrangement. They are (i) $D>>d$ and (ii) $d>>\lambda$, together referred to here as the Parallel Ray Approximation (PRA). From Figure 4, it is clear that we are justified in taking any two rays that are headed towards the same arbitrary point $\mathrm{P}$ on the screen as approximately parallel to each other in the vicinity of $S_{1}$ and $\mathrm{S}_{2}$. The value of $\delta$ determines whether the two waves from either slit, arrive at point $\mathrm{P}$ on the screen in phase or out of phase. If $\delta$ is an integer multiple of $\lambda$, then the two waves from $\mathrm{S}_{1}$ and $\mathrm{S}_{2}$ are in phase and constructive interference results. However, if $\delta$ is an odd integer multiple of $\lambda / 2$, the two waves from $\mathrm{S}_{1}$ and $\mathrm{S}_{2}$ are $180^{\circ}$ out of phase and destructive interference results.

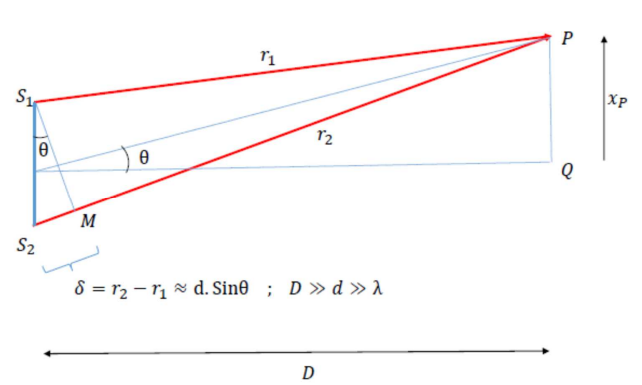

Figure 3. Conventional geometrical analysis.

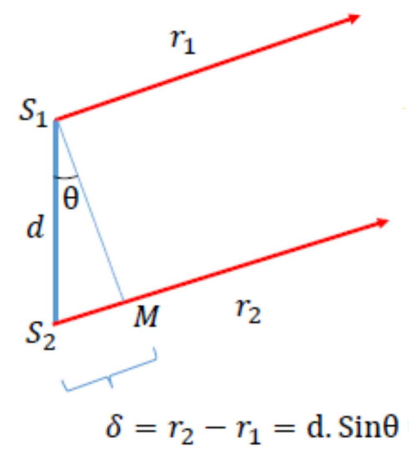

Figure 4. Parallel Ray Approximation.

Condition for Constructive Interference:

$$
\delta=d \cdot \sin \theta=n \cdot \lambda ; n=0,1,2, \ldots
$$

Condition for Destructive Interference:

$$
\delta=d \cdot \sin \theta=(2 n+1) \cdot \lambda / 2 ; n=0,1,2, \ldots
$$

Where $\mathrm{n}$ is referred to as the order of the fringe. If the PRA holds true, the angle $\theta$ is very small and we can take $\sin \theta \approx$ $\tan \theta$. From Figure 3 , it is clear that $\tan \theta=\frac{x_{P}}{D}$ where $x_{P}$ is the distance of the point $\mathrm{P}$ from the center $\mathrm{Q}$ of the distant screen. By making these substitutions into (2) and (3) we arrive at the classical results for positions of bright and dark fringes, respectively:

$$
\begin{gathered}
x_{\text {bright }}=n \cdot \frac{D \lambda}{d} \\
x_{\text {dark }}=\frac{(2 n+1)}{2} \cdot \frac{D \lambda}{d}
\end{gathered}
$$

\subsection{Failures of the Conventional Analysis}

The above highly simplified manner of approach, also referred to as far-field analysis, can be used to predict the positions of bright and dark fringes located near the center of the distant screen, with a fair degree of precision. The analysis, however, fails to account for the position of those fringes located in the peripheral portions of the screen. It also cannot be used when the screen to slit distance becomes comparable to the inter-slit distance (near-field) and when the inter-slit distance becomes comparable to the wavelength of light. In some recently published papers, a deeper treatment has been forwarded which makes use of the equation of a hyperbola as the locus of points with a given path difference. From this hyperbola equation, an asymptotic expression is approximated 
to determine the position of a fringe at an arbitrary point $\mathrm{P}$ on the distant screen. [7-10] Though these newer approaches make redundant the 200-year old use of the paradoxical PRA, none of them derive the hyperbola equation from first principles. In this paper, a theorem is stated (its proof was first forwarded in the appendix of [11] and is repeated here in §3) which will give the student a much better pictorial grasp of the underlying geometry of wave interference.

\section{The New Analysis}

\subsection{Theorem}

Using analytical geometry and differential calculus, it can be shown that the locus of the points of intersections of two uniformly expanding circular wavefronts with non-coincident point source centers $A(-a, 0)$ and $B(a, 0)$, speed of propagation $u$ and time difference of emanation of circular wavefronts $\Delta t_{A B}$, is a hyperbola (see Figure 5), whose equation is given by:

$$
\frac{x^{2}}{\left(\frac{u \Delta t_{A B}}{2}\right)^{2}}-\frac{y^{2}}{a^{2}-\left(\frac{u \Delta t_{A B}}{2}\right)^{2}}=1
$$
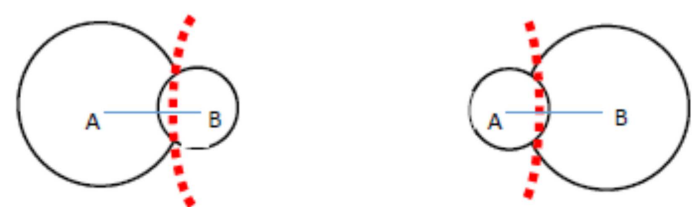

Figure 5. Locus of the points of intersections of two uniformly expanding circular wavefronts is a branch of a hyperbola (red dotted line). When source center A emanates a wavefront before $B$, the right hyperbolic branch is formed and when $B$ emanates before A, the left hyperbolic branch is formed.

\subsection{Application of the Theorem}

The above hyperbola equation can be directly applied to the experimental arrangement of the double slit apparatus, by choosing the origin $\mathrm{O}$ to lie midway between the narrow slits $\mathrm{S}_{1}$ and $\mathrm{S}_{2}$, the $\mathrm{X}$-axis to lie along $\mathrm{S}_{1} \mathrm{~S}_{2}$ and the $\mathrm{Y}$-axis to lie along the OQ direction (see Figures $1 \& 3$ ). Since the slits act as the centers of expansion of two uniformly expanding circular wavefronts of light, the parameters $\left\{x, y, u, \Delta t_{A B}, a\right\}$ may be replaced by $\left\{x_{P}, D, c, \tau, d / 2\right\}$, where $\mathrm{c}$ is the speed of light and $\tau$ is the time difference of arrival of rays $\mathrm{S}_{1} \mathrm{P}$ and $\mathrm{S}_{2} \mathrm{P}$ at an arbitrary point $\mathrm{P}$ on the screen. On making these substitutions into (6), we get:

$$
\frac{x_{P}{ }^{2}}{\left(\frac{c \cdot \tau}{2}\right)^{2}}-\frac{D^{2}}{\frac{d^{2}}{4}-\left(\frac{c \cdot \tau}{2}\right)^{2}}=1 \Rightarrow x_{P}^{2}=\left(\frac{c \cdot \tau}{2}\right)^{2}\left(1+\frac{D^{2}}{\frac{d^{2}}{4}-\left(\frac{c \cdot \tau}{2}\right)^{2}}\right)
$$

The path difference between rays $\mathrm{S}_{1} \mathrm{P}$ and $\mathrm{S}_{2} \mathrm{P}$ at an arbitrary point $\mathrm{P}$ is:

$$
\delta=S_{2} P-S_{1} P=r_{2}-r_{1}
$$

Since $\tau$ is the difference in the times of arrival of rays $\mathrm{S}_{1} \mathrm{P}$ and $\mathrm{S}_{2} \mathrm{P}$ at $\mathrm{P}$, we may write:

$$
\tau=t_{S_{2} P}-t_{S_{1} P}=\frac{S_{2} P}{c}-\frac{S_{1} P}{c}=\frac{r_{2}-r_{1}}{c}=\frac{\delta}{c} \Rightarrow \delta=c . \tau
$$

Substituting (9) in (7), we get:

$$
x_{P}^{2}=\left(\frac{\delta}{2}\right)^{2}\left(1+\frac{D^{2}}{\frac{d^{2}}{4}-\left(\frac{\delta}{2}\right)^{2}}\right) \Rightarrow x_{P}=\sqrt{\frac{\delta^{2}}{4}+\frac{D^{2} \cdot \delta^{2}}{d^{2}-\delta^{2}}}
$$

Equation (10) expresses the exact position of an interference fringe at an arbitrary point $P\left(x_{P}, D\right)$ on the screen, in terms of the path difference $\delta$, screen distance $D$ and inter-slit separation $d$. Unlike the original analysis, this new approach does not invoke the twin assumptions of the Parallel Ray Approximation. The ensuing predictions can therefore, justifiably claim precision.
Double Slit Barrier

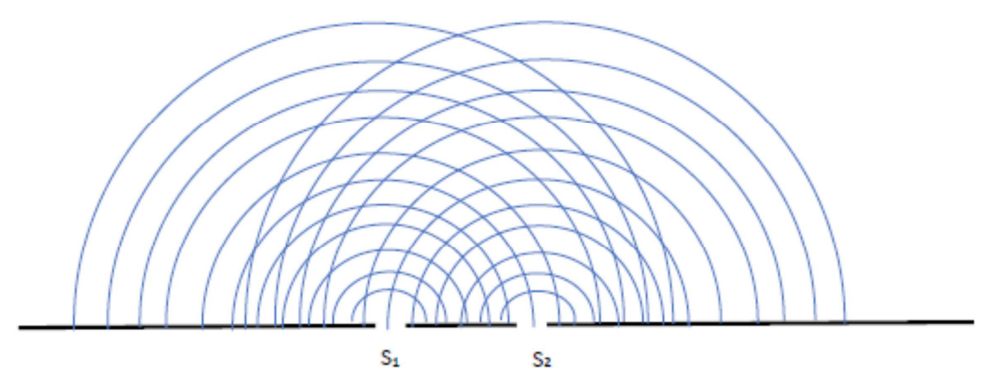

Figure 6. Circular wavefronts emanating from two slits.

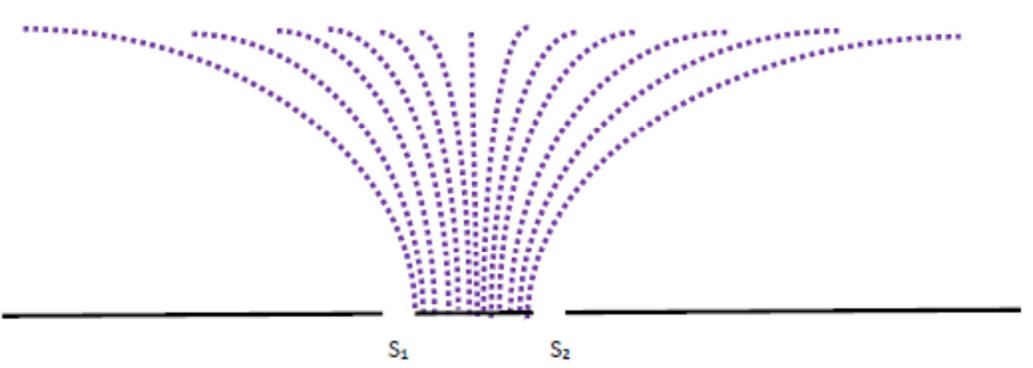




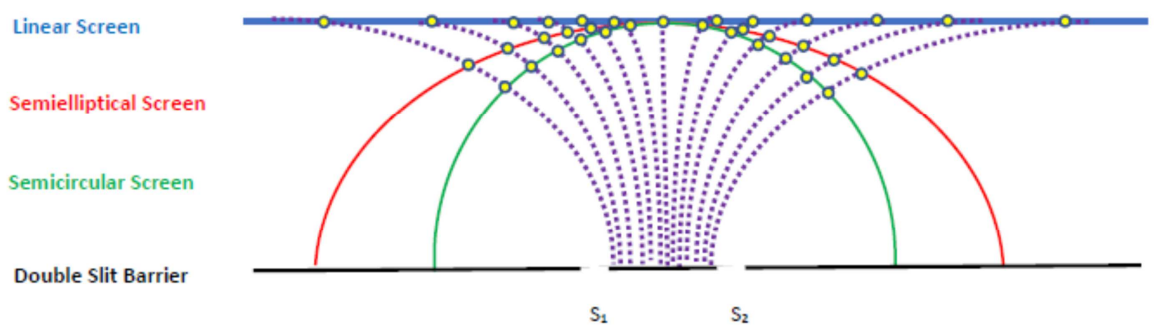

Figure 8. Interference fringes are formed at the intersection points of the hyperbolas with each type of screen.

\subsection{Further extensions of the New Analysis}

The new approach proposed in this paper can be further extended by varying the shape of the screen used to capture the fringe pattern. From Figures $6 \& 7$ it is clear that the series of circular wavefronts emanating from slits $S_{1}$ and $S_{2}$ give rise to a family of confocal hyperbolae. It is where these hyperbolae intersect with the distant screen that the interference fringes are formed. In Figure 8, the screens are varied in shape, namely, (i) linear, (ii) semielliptical, (iii) semicircular and the fringes formed on them are depicted as yellow dots. If $\mathrm{O}$ and $\mathrm{Q}$ be the midpoint of $S_{1} S_{2}$ and the screen, respectively, then the angular position of each fringe maybe calculated with respect to $\mathrm{OQ}$ as the reference line (see Figure 9). By devising such an experimental arrangement, the distribution of the fringes may be compared for each screen shape and the non-uniformity in their spacings and widths confirmed.

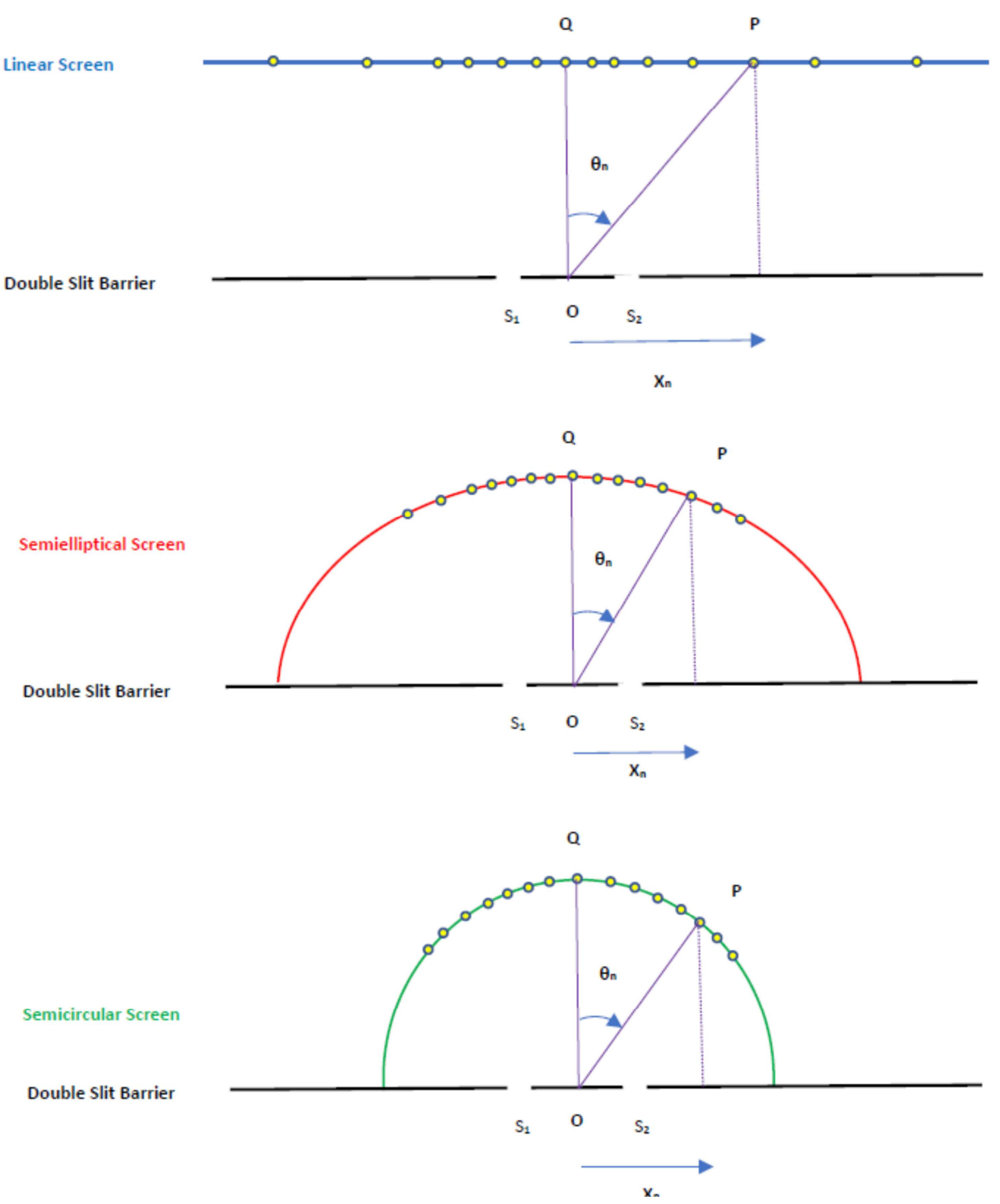

Figure 9. Angular position $\theta_{n}$ of an nth order interference fringe located at point $P$ on a linear, semielliptical and semicircular screen. 


\subsubsection{Angular Position Formula for Interference Fringes Formed on a Linear Screen}

Let the equation of the hyperbola representing the interference of the circular wavefronts be $\frac{x^{2}}{\frac{\delta^{2}}{4}}-\frac{y^{2}}{\frac{d^{2}-\delta^{2}}{4}}=1$ and the equation of the line representing the linear screen be $y=D$. Then by solving these two equations, we obtain the point locations of the interference fringes on the screen as $\left( \pm \sqrt{\frac{\delta^{2}}{4}+\frac{D^{2} \cdot \delta^{2}}{d^{2}-\delta^{2}}}, D\right)$. Also, it may be shown that the angular position for an interference fringe corresponding to some path difference $\delta$ is given by $\theta=\tan ^{-1}\left( \pm \sqrt{\frac{\delta^{2}}{4 D^{2}}+\frac{\delta^{2}}{d^{2}-\delta^{2}}}\right)$.

\subsubsection{Angular Position Formula for Interference Fringes Formed on a Semielliptical Screen}

Let the equation of the hyperbola representing the interference of the circular wavefronts be $\frac{x^{2}}{\frac{\delta^{2}}{4}}-\frac{y^{2}}{\frac{d^{2}-\delta^{2}}{4}}=1$ and the equation of the ellipse representing the semielliptical screen be $\frac{x^{2}}{E^{2}}+\frac{y^{2}}{F^{2}}=1$ where $\mathrm{E}$ and $\mathrm{F}$ are the semi-major and semi-minor axes, respectively. Then by solving these two equations, we obtain the point locations of the interference fringes on the screen as $\left( \pm \sqrt{\frac{\frac{1}{B^{2}}+\frac{1}{F^{2}}}{\frac{1}{A^{2} F^{2}}+\frac{1}{B^{2} E^{2}}}}, \sqrt{\frac{\frac{1}{A^{2}}-\frac{1}{E^{2}}}{\frac{1}{A^{2} F^{2}}+\frac{1}{B^{2} E^{2}}}}\right)$. Also, it may be shown that the angular position for an interference fringe corresponding to some path difference $\delta$ is given by $\theta=\tan ^{-1}\left( \pm\left(\frac{E}{F}\right) \cdot \sqrt{\left(\frac{\delta^{2}}{d^{2}-\delta^{2}}\right)\left(\frac{4 F^{2}+d^{2}-\delta^{2}}{4 E^{2}-\delta^{2}}\right)}\right) \cdot$ (N.B. $A^{2}=\frac{\delta^{2}}{4}$ and $B^{2}=\frac{d^{2}-\delta^{2}}{4}$ )

\subsubsection{Angular Position Formula for Interference Fringes Formed on a Semicircular Screen}

Let the equation of the hyperbola representing the interference of the circular wavefronts be $\frac{x^{2}}{\frac{\delta^{2}}{4}}-\frac{y^{2}}{\frac{d^{2}-\delta^{2}}{4}}=1$ and the equation of the circle representing the semicircular screen with radius $\mathrm{R}$ be $x^{2}+y^{2}=R^{2}$. Then by solving these two equations, we obtain the point locations of the interference fringes on the screen as $\left( \pm \sqrt{\frac{\frac{R^{2}}{B^{2}}+1}{\frac{1}{A^{2}}+\frac{1}{B^{2}}}}, \sqrt{\frac{\frac{R^{2}}{A^{2}}-1}{\frac{1}{A^{2}}+\frac{1}{B^{2}}}}\right)$. Also, it may be shown that the angular position for an interference fringe corresponding to some path difference $\delta$ is given by $\theta=\tan ^{-1}\left( \pm \sqrt{\left(\frac{\delta^{2}}{d^{2}-\delta^{2}}\right)\left(\frac{4 R^{2}+d^{2}-\delta^{2}}{4 R^{2}-\delta^{2}}\right)}\right)$. $A^{2}=\frac{\delta^{2}}{4}$ and $B^{2}=\frac{d^{2}-\delta^{2}}{4}$ )

\section{Mathematical Proof of Theorem (Equation 6)}

Consider two point sources A and B located at positions $(-a, 0)$ and $(a, 0)$, respectively in a two-dimensional
XY-plane, with the Origin $O(0,0)$ lying mid-way between them. Say that the Source A emits a circular wavefront at an instant of time $t_{A}$ and Source B emits a similar circular wavefront, at a later instant $t_{B}$. Also assume that the speed of propagation $u$ of both wavefronts is equal and uniform in all directions. Then the equation of the circular wavefront emanating from source $A(-a, 0)$, at a given time $t>t_{A}$, can be written as:

$$
(x+a)^{2}+y^{2}=R^{2}
$$

Similarly, the equation of the circular wavefront emanating from source $B(a, 0)$, at the instant $t>t_{B}$, can be written as:

$$
(x-a)^{2}+y^{2}=r^{2}
$$

Where $\mathrm{R}$ and $\mathrm{r}$ are the instantaneous radii of the wavefronts emanating from sources $\mathrm{A}$ and $\mathrm{B}$, respectively. Note that, $R>r$ for $t_{A}<t_{B}$. Recall that the speed of propagation of both wavefronts $u$ is equal and uniform in all directions, given by:

$$
u=\frac{d R}{d t}=\frac{d r}{d t}
$$

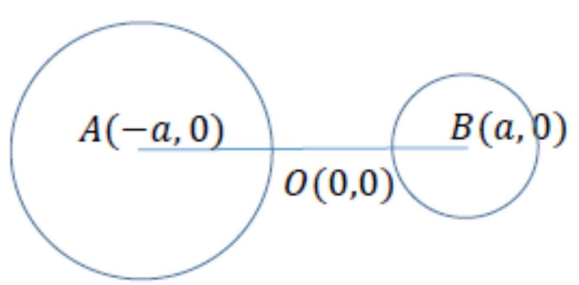

Figure 10. Sources $A$ and $B$ emitting circular wavefronts in temporal succession.

Subtracting (12) from (11),

$$
(x+a)^{2}-(x-a)^{2}=R^{2}-r^{2}
$$

On simplifying,

$$
x=\frac{\left(R^{2}-r^{2}\right)}{4 a}
$$

Squaring (14),

$$
x^{2}=\frac{\left(R^{2}-r^{2}\right)^{2}}{16 a^{2}}
$$

Differentiating (15) with respect to time

$$
2 x \frac{d x}{d t}=\frac{2\left(R^{2}-r^{2}\right)\left(2 R \cdot \frac{d R}{d t}-2 r \cdot \frac{d r}{d t}\right)}{16 a^{2}}
$$

Substituting (13) in the above, we get,

$$
\begin{gathered}
2 x \frac{d x}{d t}=\frac{4 u\left(R^{2}-r^{2}\right)(R-r)}{16 a^{2}} \\
2 x \frac{d x}{d t}=\frac{4 u(R+r)(R-r)^{2}}{16 a^{2}}
\end{gathered}
$$

Substituting (14) in (11),

$$
y^{2}=R^{2}-(x+a)^{2}
$$




$$
\begin{gathered}
=R^{2}-\left(\frac{\left(R^{2}-r^{2}\right)}{4 a}+a\right)^{2} \\
=\left(R+\left(\frac{\left(R^{2}-r^{2}\right)}{4 a}+a\right)\right)\left(R-\left(\frac{\left(R^{2}-r^{2}\right)}{4 a}+a\right)\right) \\
=\frac{\left(R^{2}-r^{2}+4 a^{2}+4 a R\right) \cdot\left(-R^{2}+r^{2}-4 a^{2}+4 a R\right)}{16 a^{2}} \\
=-\frac{\left(R^{4}+r^{4}+16 a^{4}-2 R^{2} r^{2}-8 a^{2} R^{2}-8 a^{2} r^{2}\right)}{16 a^{2}} \\
=-\frac{\left[\left(R^{2}+r^{2}-4 a^{2}\right)^{2}-4 R^{2} r^{2}\right]}{16 a^{2}} \\
=-\frac{\left[\left((R-r)^{2}+2 R r-4 a^{2}\right)^{2}-4 R^{2} r^{2}\right]}{16 a^{2}} \\
=-\frac{\left[\left((R-r)^{2}+2 R r-4 a^{2}\right)+2 R r\right]\left[\left((R-r)^{2}+2 R r-4 a^{2}\right)-2 R r\right]}{16 a^{2}} \\
=-\frac{\left((R-r)^{2}+4 R r-4 a^{2}\right)\left((R-r)^{2}-4 a^{2}\right)}{16 a^{2}} \\
y^{2}=-\frac{\left((R+r)^{2}-4 a^{2}\right)\left((R-r)^{2}-4 a^{2}\right)}{16 a^{2}}
\end{gathered}
$$

From (17), it is clear that in order for $y \in \mathbb{R}$ either one of the following two conditions must hold true:

(i) $R+r>2 a$ and $R-r<2 a$, or

(ii) $R+r<2 a$ and $R-r>2 a$

In order that the two circular wavefronts intersect each other to trace out the locus of some curve, (it will be later shown that the curve is a branch of a hyperbola with vertex $\mathrm{V}$ lying somewhere on the line $\mathrm{AB}$ joining the point sources $\mathrm{A}$ and $\mathrm{B}$ ), it is necessary that condition (i) holds true. Condition (ii) would geometrically imply that the circles intersect nowhere in the XY-plane and is therefore rejected. So provided condition (i) holds true, we can write:

$$
y= \pm \sqrt{-\frac{\left((R+r)^{2}-4 a^{2}\right)\left((R-r)^{2}-4 a^{2}\right)}{16 a^{2}}} \in \mathbb{R}
$$

Differentiating (17) with respect to time,

$2 y \cdot \frac{d y}{d t}=-\frac{\left[\left((R+r)^{2}-4 a^{2}\right) \cdot 2(R-r)\left(\frac{d R}{d t}-\frac{d r}{d t}\right)+\left((R-r)^{2}-4 a^{2}\right) \cdot 2(R+r)\left(\frac{d R}{d t}+\frac{d r}{d t}\right)\right]}{16 a^{2}}$

Substituting (13) in the above, we get,

$$
2 y \cdot \frac{d y}{d t}=-\frac{4 u(R+r)\left((R-r)^{2}-4 a^{2}\right)}{16 a^{2}}
$$

To re-iterate, $t_{A}$ and $t_{B}$ are the instants at which the sources $\mathrm{A}$ and $\mathrm{B}$ emit circular wavefronts, respectively $\left(t_{A}<t_{B}\right)$. Additionally, let us assume $\tau$ to be the instant at which both these expanding wavefronts come to meet at a common point $\mathrm{V}$ lying on the line $\mathrm{AB}$ (see Figure 11). We can therefore reason that the wavefront arising from source A, would have grown from an initial radius $R=0$ to $R=$ $R(\tau)$ in the time interval spanning $t_{A}$ to $\tau$. Similarly, the wavefront arising from source $B$, would have grown from an initial radius $r=0$ to $r=r(\tau)$ in the time interval spanning $t_{B}$ to $\tau$. So, it should be possible to integrate equation (13), keeping in mind that the speed of propagation of both wavefronts is equal and uniform in all directions and that $t_{A}<t_{B}<\tau$ :

$$
\begin{aligned}
& \int_{0}^{R(\tau)} d R=\int_{t_{A}}^{\tau} u . d t \Rightarrow R(\tau)=u\left(\tau-t_{A}\right) \\
& \int_{0}^{r(\tau)} d r=\int_{t_{B}}^{\tau} u \cdot d t \Rightarrow r(\tau)=u\left(\tau-t_{B}\right)
\end{aligned}
$$

At the instant, $t=\tau$, both wavefronts meet at the point $\mathrm{V}$ on the line $A B=2 a$. So, we can write,

$$
R(\tau)+r(\tau)=2 a
$$

Subtracting (21) from (20),

$$
R(\tau)-r(\tau)=u\left(t_{B}-t_{A}\right)=u . \Delta t_{A B}
$$

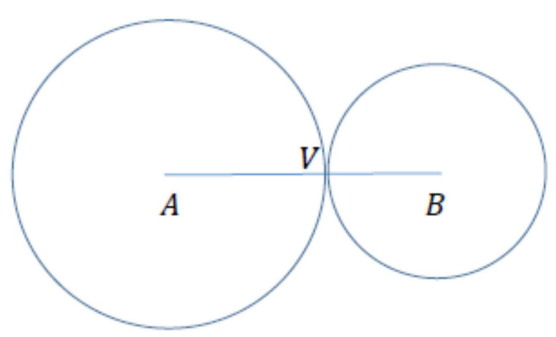

Figure 11. Circular wavefronts expand to meet at a single point Vlying on the line joining $A$ and $B$.

The two expanding circular wavefronts will intersect each other at two points, call them $\mathrm{P}$ and $\mathrm{P}$ ', after time $t>\tau$ (see Figure 12). The $(x, y)$ co-ordinates of these point-pair intersections are given by equations (14) and (18):

$$
\left(\frac{\left(R^{2}-r^{2}\right)}{4 a}, \pm \sqrt{-\frac{\left((R(t)+r(t))^{2}-4 a^{2}\right)\left((R(t)-r(t))^{2}-4 a^{2}\right)}{16 a^{2}}}\right)
$$

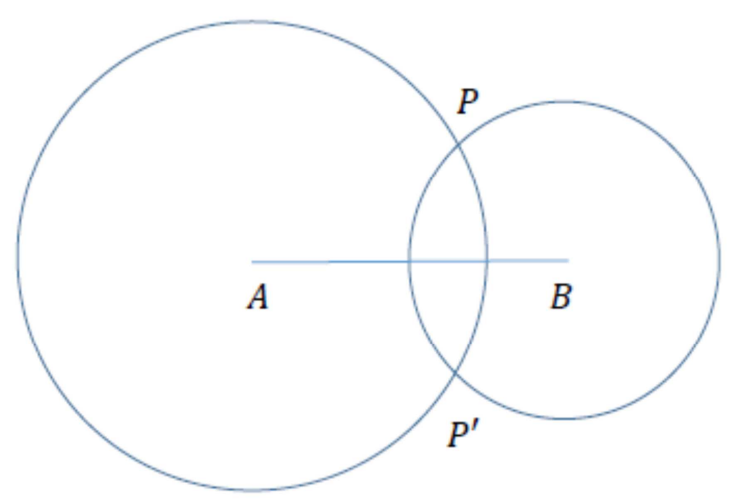

Figure 12. Circular wavefronts expand to intersect each other at two points $P$ and $P$ '.

The co-ordinate of the point $\mathrm{V}$ lying on $\mathrm{AB}$ can be found by substituting (22) \& (23) in (24):

$$
\left(\frac{u \Delta t_{A B}}{2}, 0\right)
$$

Since the two circular wavefronts propagate outwards at the same expansion rate $u$, we can expect that the instantaneous 
difference in their radii, $R(t)-r(t)$ to be constant with time. A formal justification of this statement can be made as follows:

$$
\begin{gathered}
\frac{d(R(t)-r(t))}{d t}=\frac{d R}{d t}-\frac{d r}{d t}=u-u=0(\mathrm{By}(13)) \\
\Rightarrow R(t)-r(t)=\text { constant }
\end{gathered}
$$

This would imply that Equation (23) should hold true for all times, $t \geq \tau$. That is,

$$
R(t)-r(t)=u\left(t_{B}-t_{A}\right)=u . \Delta t_{A B}
$$

This satisfies the defining property of a hyperbola, as the locus of the point whose difference in the distances from two fixed points (foci), is a constant. That implies, the locus of the point of intersections of two circular wavefronts emanating from sources A and B, takes the shape of a hyperbola, since the differences in their instantaneous radii have been shown to be constant. Therefore, $V\left(\frac{u \Delta t_{A B}}{2}, 0\right)$ will be the co-ordinate of the vertex of one branch of a hyperbola, generated when source A emits a circular wavefront before source B. The vertex of the complementary branch of the hyperbola is generated when source $B$ emits a circular wavefront before source $A$ and has its vertex at the co-ordinate $V^{\prime}\left(-\frac{u \Delta t_{B A}}{2}, 0\right)$, since $\Delta t_{A B}=t_{B}-t_{A}=-\left(t_{A}-t_{B}\right)=-\Delta t_{B A}$.

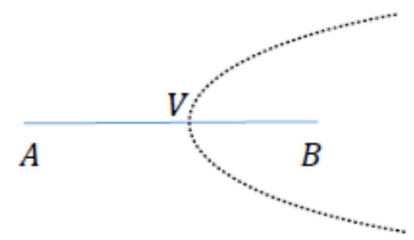

Figure 13. Locus of the Intersection Points when Source A emits before Source B.

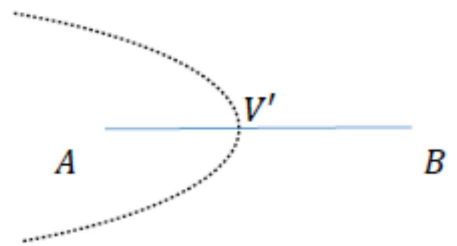

Figure 14. Locus of the Intersection Points when Source B emits before Source A.

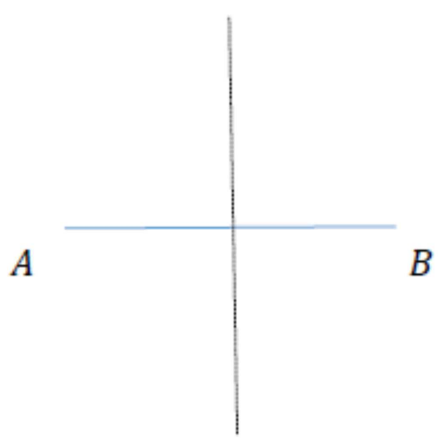

Figure 15. Locus of the Intersection Points when Sources $A$ and $B$ emit simultaneously.
The general equation of a hyperbola with center at origin and transverse axis along the $\mathrm{X}$-axis is:

$$
\frac{x^{2}}{C^{2}}-\frac{y^{2}}{D^{2}}=1
$$

Where $C$ and $D$ are the semi-lengths of the transverse and conjugate axes respectively. The value of the constant $C$ is already known to us from (25) since it represents the distance of the vertex of the hyperbola from the origin. That is,

$$
C=\frac{u \Delta t_{A B}}{2}
$$

However, the value of the constant $D$ is yet to be determined. Once $D$ is found and put into (27), we would have arrived at the required equation of the hyperbola. (Note that the sources $A(-a, 0)$ and $B(a, 0)$ lie at the foci of the hyperbola).

Differentiating Equation (27) with respect to time:

$$
\frac{1}{C^{2}} 2 x \frac{d x}{d t}-\frac{1}{D^{2}} 2 y \frac{d y}{d t}=0
$$

The above equation should hold true for all times $t \geq \tau>$ $t_{B}>t_{A}$. This would mean that for $t=\tau$,

$$
\frac{1}{C^{2}} \cdot 2 x \frac{d x}{d t}_{t=\tau}-\frac{1}{D^{2}} \cdot 2 y \frac{d y}{d t}_{t=\tau}=0
$$

From Equations (16), (22) and (23),

$$
\begin{aligned}
& 2 x \frac{d x}{d t}_{t=\tau}=\frac{4 u(R(\tau)+r(\tau))(R(\tau)-r(\tau))^{2}}{16 a^{2}} \\
&=4 u \cdot 2 a \cdot \frac{\left(u \Delta t_{A B}\right)^{2}}{16 a^{2}} \\
& 2 x \frac{d x}{d t}_{t=\tau}=\frac{u^{3}\left(\Delta t_{A B}\right)^{2}}{2 a}
\end{aligned}
$$

From Equations (19), (22) and (23),

$$
\begin{aligned}
& 2 y \cdot \frac{d y}{d t} t_{t=\tau}=-\frac{4 u(R(\tau)+r(\tau))\left((R(\tau)-r(\tau))^{2}-4 a^{2}\right)}{16 a^{2}} \\
& 2 y \cdot \frac{d y}{d t}_{t=\tau}=-4 u \cdot \frac{2 a\left(\left(u \cdot \Delta t_{A B}\right)^{2}-4 a^{2}\right)}{16 a^{2}} \\
& 2 y \cdot \frac{d y}{d t}_{t=\tau}=-\frac{u\left(\left(u \cdot \Delta t_{A B}\right)^{2}-4 a^{2}\right)}{2 a}
\end{aligned}
$$

Substituting (30), (31) and (28) in Equation (29),

$$
\frac{1}{\left(\frac{u \Delta t_{A B}}{2}\right)^{2}} \frac{u^{3}\left(\Delta t_{A B}\right)^{2}}{2 a}-\frac{1}{D^{2}}\left(-\frac{u\left(\left(u . \Delta t_{A B}\right)^{2}-4 a^{2}\right)}{2 a}\right)=0
$$

On algebraic simplification of the above, we get:

$$
D^{2}=a^{2}-\frac{u^{2}\left(\Delta t_{A B}\right)^{2}}{4}=a^{2}-\left(\frac{u \Delta t_{A B}}{2}\right)^{2}=a^{2}-C^{2}
$$

Substituting (28) and (32) in (27), we finally arrive at, 


$$
\frac{x^{2}}{\left(\frac{u \Delta t_{A B}}{2}\right)^{2}}-\frac{y^{2}}{a^{2}-\left(\frac{u \Delta t_{A B}}{2}\right)^{2}}=1
$$

This is the analytical equation of the hyperbola representing the locus of all the points of intersection between two circular wavefronts emanating from sources $\mathrm{A}$ and $\mathrm{B}$, emitted at times $t_{A}$ and $t_{B}$, respectively $\left(t_{A}<t_{B}\right)$. It is expressed in terms of the Inter-Source Interval $\Delta t_{A B}$, the speed of propagation of the circular wavefront $u$ and the position of the sources $( \pm a, 0)$ with respect to the origin $\mathrm{O}$, which lies midway between them.

\section{Conclusion: Some Remarks on the New Analysis}

\subsection{Reduction to the Conventional Results}

If the PRA assumptions are taken into account (i.e. D $>>d$ and $\mathrm{d}>>\lambda$ ), then the $\delta$ terms in equation (10) can be neglected and it would reduce right back to the classical results (4) and (5) for constructive and destructive interference, respectively. The new analysis can therefore, be considered as a generalization of the old, wherein no assumptions are invoked and a set of exact predictions for fringe position are furnished.

$$
x_{P}=\sqrt{0+\frac{D^{2} \cdot \delta^{2}}{d^{2}-0}}=\sqrt{\frac{D^{2} \cdot \delta^{2}}{d^{2}}}=\frac{D \cdot \delta}{d}
$$

\subsection{Distribution of Fringes}

According to the old analysis, the fringes are of equal width and are spaced equally apart. However, from the new analysis it is clear that the fringes are of unequal widths and are unequally spaced apart. Infact, the fringes near the center of the screen are narrower and more crowded together while those in the periphery of the screen are wider and more spread out. These predictions will become more evident as the inter-slit distance approaches the wavelength of light used. Though this may be difficult to achieve using visible light $(430 \mathrm{THz}-770 \mathrm{THz})$ owing to the very small wavelengths involved, it may be more readily demonstrated using microwaves $(300 \mathrm{MHz}-300 \mathrm{GHz})$.

\subsection{Pedagogic Advantages}

The theorem forwarded in this paper will give the student a much better insight and pictorial grasp of the underlying geometry of wave interference, that is applicable to both the far field and the near field scenarios. It also, completely discards the use of the parallel ray approximation and is therefore, a more generalized approach. For these reasons, the author suggests that the new analysis ought to be introduced into the physics curriculum at both the undergraduate and the Senior High School levels, to replace the conventional approach. On a philosophical note, it also serves to instruct the student of the importance of viewing every existing theoretical and experimental method in physics with a critical eye, regardless of how many centuries of acclaim and renown it may have enjoyed. This attitude indeed, encapsulates the true spirit of all scientific progress and in the context of Young's 200-year old experiment, Daniel Meyer gives a good polemic on the subject. [12]

\subsection{Generalization of the New Analysis to N-slit Interference and Diffraction}

The theorem forwarded in this paper was derived for the special case wherein there are only two very narrow slit sources involved. The next logical step is to generalize the hyperbola equation for $\mathrm{N}$ such equally spaced slits. It should then be possible to study the phenomenon of diffraction by setting $\mathrm{N} \rightarrow \infty$ and/or $\mathrm{d} \rightarrow 0$. This project is currently underway by the author and presented below is one of the preliminary results obtained, named the Generalized Hyperbola Equation for any slit pair $i$ and $j$ of $\mathrm{N}$ equally spaced slits:

$$
\frac{(x-\mu d)^{2}}{\left(\frac{\delta_{i j}}{2}\right)^{2}}-\frac{y^{2}}{\left(\frac{v d}{2}\right)^{2}-\left(\frac{\delta_{i j}}{2}\right)^{2}}=1
$$

Here, $i$ and $j$ represents the slit source number; $i=\{1,2,3, \ldots, N\} ; j=\{1,2,3, \ldots, N\} ; j>i ; i \neq j ; \mu=\frac{i+j-3}{2}$; $v=j-i ; \delta_{i j}$ is the path difference for rays from either slit; $d$ is the inter-slit spacing. The above generalized hyperbola equation can be shown to reduce to equation (10) which is the formula for two-slit interference when $i=1$ and $j=2$.

\subsection{From Classical to Quantum Physics}

As a final note, the author asserts that the hyperbola theorem stated and derived herein may find some suitable application in the Pilot Wave interpretation of Quantum Mechanics, which was first proposed by Louis de Broglie and then later taken up by David Bohm. [13, 14, 15] It is suggested that the experts working in the field of Bohmian Mechanics consider taking up this research proposal.

\section{Acknowledgements}

Gloria in Excelsis Deo

I wish to thank my parents, Dr. Shweta, Dr. Joe, Prof. Sisir Roy (NIAS), Prof. SP Basavaraju (VTU), Prof. RI Sujith (IIT-M), Mr. Shoukat Ali sir (IHS), Pastor Johnson (Bethel AG), Mr. KK Kutty (IGNOU), Gabriel and my department colleagues at East Point Medical College, for all the love, encouragement and support they've showered upon me during the preparation of this manuscript.

\section{References}

[1] Young, T. (1804). I. The Bakerian Lecture. Experiments and calculations relative to physical optics. Philosophical transactions of the Royal Society of London, 94, 1-16.

[2] Young, T. (1807). A Course of Lectures on Natural Philosophy and the Mechanical Arts, Vol. 1 (William Savage, Bedford Bury, London), pp. 463-465. 
[3] Halliday, Resnick and Walker, Fundamentals of Physics, 6th Edition (John Wiley and Sons, Inc., New York, 2003) pp. 866-870.

[4] Giancoli, Physics for Scientists \& Engineers, 4th Edition (Pearson- Prentice Hall, New Jersey, 2008) pp. 903-909.

[5] Young and Freedman, Sears and Zemansky's University Physics, 13th Edition (Peason Education publishing as Addison-Wesley, San Francisco CA, 2012) pp. 1164-1170.

[6] Born and Wolf. Principles of optics: electromagnetic theory of propagation, interference and diffraction of light, $4^{\text {th }}$ Edition (Elsevier, 2013) pp. 256-260.

[7] Sobel, Michael I. "Algebraic Treatment of Two-Slit Interference." The Physics Teacher 40, no. 7 (2002): 402-404.

[8] Poon, Dick CH. "How Good Is the Approximation "Path Difference $\approx \mathrm{d} \sin \theta$ "?." The Physics Teacher 40, no. 8 (2002): 460-462.

[9] Hopper, Seth, and John Howell. "An exact algebraic evaluation of path-length difference for two-source interference." The Physics Teacher 44, no. 8 (2006): 516-520.
[10] Hughes, Joe, and Frederic Liebrand. "Conic Sections in the Double-Slit Experiment."

[11] Thomas, J. "A Mathematical Treatise on Polychronous Wavefront Computation and its Application into Modeling Neurosensory Systems. Research Gate; 2014." Unpublished.

[12] Meyer, Daniel Z. "A Student-Centered, Inquiry-Based Approach to Young's Double-Slit Experiment (and Other Investigations of Light's Wave Character)." The Physics Teacher 55, no. 3 (2017): 159-163.

[13] de Broglie, Louis. "Wave mechanics and the atomic structure of matter and radiation." Le Journal de Physique et le Radium8 (1927): 225.

[14] Böhm, David. "A Suggested Interpretation of the Quantum Theory in Terms of" Hidden" Variables. I." Physical Review 85, no. 2 (1952): 166-179.

[15] Böhm, David. "A Suggested Interpretation of the Quantum Theory in Terms of" Hidden" Variables. II." Physical Review 85, no. 2 (1952): 180-193. 